\title{
Artemita bicolor Kertész, novo sinônimo de Artemita podexargenteus Enderlein, (Diptera, Stratiomyidae) com notas nas terminálias masculina e feminina
}

\author{
Alexandre Ururahy-Rodrigues \\ Departamento de Biologia Animal, Instituto de Biologia, Universidade Federal Rural do Rio de Janeiro. Rodovia BR 465, $\mathrm{km}$ \\ 07, 23890-000 Seropédica, Rio de Janeiro, Brasil. E-mail: ururahybioent@ig.com.br
}

\begin{abstract}
Artemita bicolor Kertész, new synonym of Artemita podexargenteus Enderlein,(Diptera, Stratiomyidae) with notes on male and female terminalia. The Stratiomyidae genus Artemita Walker, 1854 is represented in the Neotropical Region by 14 species, 6 of which occur in Brazil. Despite of the important revisions by KerTész (1914) and JAMES (1971) knowledge of morphological variation within the group is rudimentary, mainly with respect to the terminalia. In this work, Artemita bicolor Kertész, 1914 is proposed as a junior synonym of Artemita podexargenteus Enderlein, 1914 and the latter is redescribed based on terminalia morphology.

KEY WORDS. Neotropical, Pachygastrinae, taxonomy.
\end{abstract}

RESUMO. O gênero de Stratiomyidae, Artemita Walker, 1854 esta representado na região Neotropical por 14 espécies, seis das quais ocorrem no Brasil. Apesar das importantes revisões de Kertész (1914) e James (1971) o conhecimento sobre a variação morfológica no grupo ainda é rudimentar, principalmente com relação a terminália. Neste trabalho, com base na morfologia da terminália Artemita bicolor Kertész, 1914 é proposta como sinônimo júnior de Artemita podexargenteus Enderlein, 1914 e a última é redescrita.

PALAVRAS CHAVE. Neotropical, Pachygastrinae, taxonomia.

WoodLey (2001) chamou atenção sobre a possibilidade de formação de "grupos naturais" na subfamília Pachygastrinae, definindo-os arbitrariamente como: "Pequenos Pachygastrinae" - moscas geralmente pretas, metálicas e com antenas aristadas e os "Grandes Pachygastrinae" - com coloração bastante variada e antenas com estilo o que o autor considera como caráter plesiomórfico. Neste último caso, o autor cita como exemplo o gênero Artemita Walker, 1854, entre outros.

Artemita Walker, 1854 representa um dos mais numerosos da subfamília. São reconhecidas atualmente 16 espécies nominais (Woodley 2001) e está representado na região Neotropical por 15 espécies.

Dentre as contribuições ao estudo do gênero, destacamse as revisões de KerTész (1914), para as espécies com distribuição mundial, e de JAmEs (1971), para as espécies da América do Sul. Estes autores procuraram segregar as espécies com base nos padrões cromáticos. Apesar da importância da morfologia de terminália como caráter taxonômico, uma única tentativa de estudo mais minuciosa incluindo a terminália do macho, foi feita por JAMEs (1971) com A. latifrons, mas o autor não apresentou nenhuma comparação com outras espécies do gênero.

James (1971) sugeriu que A. podexargenteus Enderlein, 1914 e A. bicolor Kertész, 1914 poderiam ser consideradas "possivelmente" como sinônimas com base em um estudo das variações cromáticas do tórax. Entretanto, considerou as duas espé- cies válidas em seu catálogo (JAMES 1973), o que foi ratificado por Woodley (2001).

A presente proposta de sinonímia tem como base o estudo morfológico de terminálias de machos e fêmeas.

\section{MATERIAL E MÉTODOS}

Foram examinaodos 74 exemplares (55 A. bicolor e 19 A. podexargenteus - 72 exemplares da coleção do MZSP; um exemplar da coleção do MNRJ e um exemplar da coleção do DZUP).

Para os estudos de morfologia comparada de terminália foram dissecados dois machos e cinco fêmeas de $A$. podexargenteus; dois machos e duas fêmeas de A. bicolor. As técnicas de disecção e diafanização para estudos de terminália, foram as mesmas utilizadas por UruRahy-Rodrigues et al. (2000).

Após a observação e desenho, componentes das terminálias masculina e feminina foram montados em lâminas permanentes com bálsamo do Canadá ou acondicionados em pequenos tubos do tipo "microvial" com glicerina, etiquetados e anexados aos exemplares.

A terminologia empregada nas redescrições seguiram McAlpine (1981), as descrições dos componentes da furca genital e das espermatecas das fêmeas foram as propostas por Woodley (1987) e Ururahy-Rodrigues et al. (2000).

A lista sinonímica das espécies envolvidas foi modificada de Woodley (2001).

Revista Brasileira de Zoologia 21 (2): 397-402, junho 2004 
Abreviaturas das Instituições onde estão depositados os espécimes citados: (HNHM) Zoological Department, Hungarian Natural History Museum, Budapest, Hungary; (MNRJ) Museu Nacional da Universidade Federal do Rio de Janeiro; (MZSP) Museu de Zoologia da Universidade de São Paulo; (PAN) Museum of the Institute of Zoology, Polish Academy of Science, Warsaw, Poland; (DZUP) Coleção de Entomologia Pe. Jesus Santiago Moure, Departamento de Zoologia, Universidade Federal do Paraná, Curitiba, Paraná.

\section{RESULTADOS}

\section{Artemita Walker, 1854}

Clitellaria (Artemita) Walker, 1854: 61.- Kertész, 1914: 456-462 (rev).- James, 1971: 59-70 (rev.), 1973: 50 (cat.).- Woodley, 2001: 83 (cat.).

Artemida [sic]; Loew, 1860: 75.- James, 1973: 50 (cat.).Woodley, 2001: 83 (cat.).

Hypocerina Bigot, 1879: 207, Nomen nudum (ICZN artigos 12 e 16). - James, 1973: 50 (cat.).- Woodley, 2001: 83 (cat.).

Artemita; Brauer, 1882: 86; espécie tipo: Clitellaria amenides Walker 1849: 523.- James, 1973: 50

(cat.).- Woodley, 2001: 83 (cat.).

Psegmomma Enderlein, 1914: 304; espécie tipo: Artemita argentea Osten Sacken, por designação original.- James, 1973: 50 (cat.).- Woodley, 2001: 83 (cat.).

\section{Artemita podexargenteus Enderlein, 1914}

Artemita podexargenteus Enderlein 1914: 304; holótipo fêmea, Brasil: Santa Catarina [PAN].- Kertész, 1914: 485.- Lindner, 1928: 244.- James, 1971: 68, 1973: 50 (cat).- Woodley, 2001: 85 (cat).

Artemita bicolor Kertész, 1914: 482; síntipos 1 macho: Bolívia; Coroico [HMHN, destruido] e 1 fêmea: Brasil; Espírito Santo [HMHN].- James, 1967: 98, 1971: 68, 1973: 50 (cat).James et al.,1980: 11.- Woodley, 2001: 84 (cat). Syn. nov.

Redescrição. Macho: comprimento total: 4,6-7,4 mm.

Cabeça (Figs 1-3): semi-esférica em vista dorsal, ligeiramente mais larga do que a distância entre os lobos póspronotais; olhos grandes castanho avermelhados e com densos pêlos prateados, mais abundantes na metade superior (Figs 1-3); occipício proeminente, alcançando a margem posterior do triângulo ocelar em vista lateral (Figs 2-3); triângulo ocelar castanho-escuro, ocelos grandes, sendo o anterior maior (Figs 1-2); fronte estreita, suavemente convergente no sentido da face, placas fronto-orbitais proeminentes, castanho-amareladas e cobertas de pêlos prateados (Fig. 1); face gibosa, castanho-clara e glabra (Figs 1, 3); antenas com dez segmentos, escapo e pedicelo castanho-claros com poucos e pequenos pêlos pretos; flagelômeros mais escurecidos, sendo o primeiro, maior que os demais e o oitavo flagelômero clavado, com pequenas cerdas apicais pretas (Fig. 1); palpos castanho escuros com dois segmentos, o primeiro, cerca de duas vezes menor o segundo, com grandes pêlos da mesma cor; gena com coloração e pilosidade semelhantes às placas fronto-orbitais (Fig. 1).

Tórax: preto, inflado, subtriangular em vista dorsal (Fig. 12); mesonoto com sete faixas formadas por pêlos que variam de coloração, das quais, quatro são fusionadas, faixas dorsocentrais fusionadas às humerais; pós-humerais fusionadas às préalares e dorso-centrais posteriores livres, todas prateadas, douradas, ou ambas; faixa mediana e as dorsais pretas, bronze ou com ambas (Fig. 12); escutelo formando um ângulo obtuso em relação ao tórax em vista lateral, com diminutos pêlos pretos exceto pela margem posterior que tem pêlos maiores prateados, dourados ou ambos e armado com quatro espinhos recurvados para cima, sendo os dois laterais divergentes e os dois medianos sub-paralelos; asas com nervação característica de Pachygastrinae, hialinas, exceto por áreas micropilosas castanho-escuras causando escurecimento do pterostigma e das células $\mathrm{r} 4$, $\mathrm{r} 5$ e $\mathrm{m} 1$ com a forma de uma faixa transversal; células br e bm inconspícuas; cup presente; dm pequena, sub-trapezoidal e de localização mediana; alteres alaranjados; pernas castanho-escuras com diminutos pêlos da mesma cor exceto pelos fêmures, com o ápice castanho-claro e pêlos maiores prateados.

Abdome: semicircular em vista dorsal, com cinco segmentos visíveis e coberto com diminutos pêlos pretos, exceto, por uma mancha formada por pilosidade prateada, dourada ou com ambas, estendo-se do primeiro ao quinto tergitos; tegumento preto exceto por duas manchas elípticas, avermelhadas nas margens laterais do primeiro, segundo e terceiro tergitos.

Terminália: castanha escura, com o epândrio semicircular com poucas cerdas; décimo tergito com dois cercos com forma sub-elíptica (Fig. 4); hipândrio fusionado aos gonocoxitos (Figs 5-7); gonocoxitos em vista dorsal com os apódemas gonocoxais delgados e longos, na margem posterior, com duas projeções dentiformes ligeiramente voltadas para o interior (Fig. 5); hipândrio com guia edeagal longa e escavada (Fig. 5); gonóstilos, em vista ventral, elipsóides, achatados, com três tufos de cerdas, um anterior, um lateral e um posterior (Fig. 6); edeago com apódema de forma acuminada e ápice bífido (Fig. 8); apódema ejaculador com inserção mediana e dirigido para região anterior, formando um ângulo agudo com o edeago (Fig. 9).

Fêmea. (habitus, Fig. 10): comprimento total: 4,7-7,7 mm.

Cabeça: como no macho; olhos grandes, castanhoavermelhados e com densos pelos prateados principalmente na metade superior (Figs 13-15); fronte castanha, glabra, mais larga do que no macho, com as margens laterais sub-paralelas, com as placas fronto-orbitais castanho-amareladas e cobertas de pilosidade prateada; face gibosa, castanho-clara e glabra (Fig. 13); antenas como no macho (Fig. 11).

Tórax: como no macho (Figs 10, 12).

Abdome: como no macho (Fig. 10); pós-abdome com ovipositor membranoso, castanho-claro e telescopado a partir do sexto segmento abdominal.

Terminália: em vista dorsal com o oitavo tergito com a 

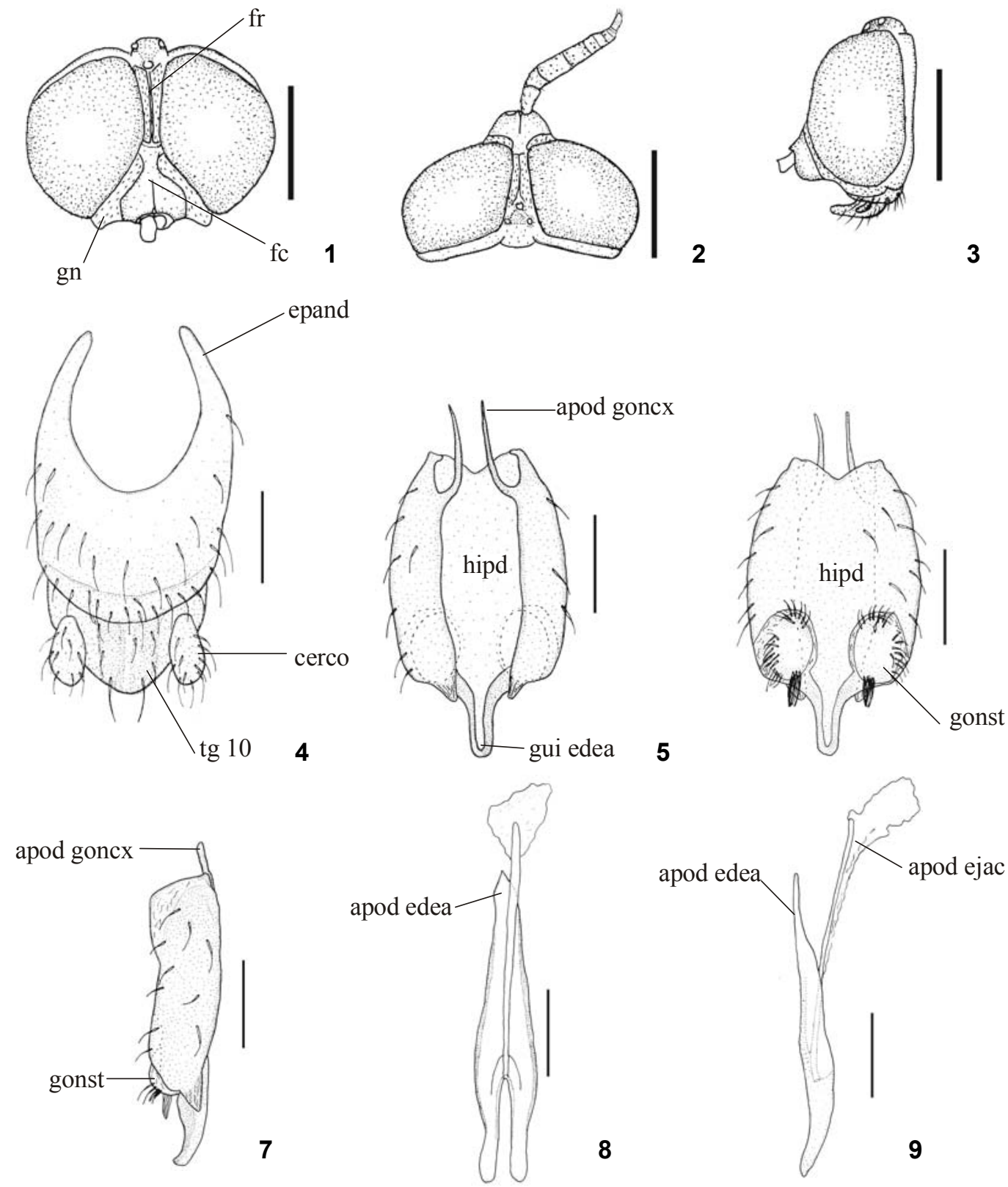

5

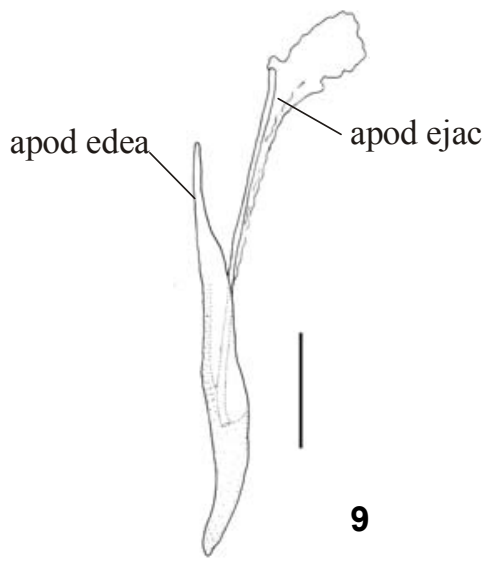

Figuras 1-9. Artemita podexargenteus macho. (1-3) Cabeça: (1) vista frontal; (2) vista dorsal; (3) vista lateral; (4-9) terminália: (4) epândrio, décimo tergito e cercos; (5-7) hipândrio, gonocoxitos e gonóstilos; (5) vista dorsal; (6) vista ventral; (7) vista lateral; (8-9) edeago; (8) vista dorsal; (9) vista lateral.(apod edea) Apódema edeagal, (apod goncx) apódema conocoxal, (apod ejac) apódema ejaculador, (epand) epândrio, (fc) face, (fr) fronte, (gn) gena, (gonst) gonóstilo, (gui edea) guia edeagal, (hipd) hipândrio, (tg 10) décimo tergito. Escalas: $1-3=1,0 \mathrm{~mm} ; 4-9=0,2 \mathrm{~mm}$.

margem posterior em forma de "W"; laterais do oitavo esternito visíveis e com diminutos pêlos castanhos escuros; nono tergito semi-retangular; décimo tergito semitriangular; cercos com dois segmentos sub-cilíndricos e do mesmo tamanho (Fig. 16); cerdas de porte médio castanhas escuras presentes no oitavo, nono, décimo tergitos e nos cercos; furca fortemente revestida por membrana, com processo anterior com forma acuminada, com duas aberturas, a primeira anterior, maior e piriforme em vista dorsal, a segunda, mediana com forma semi-elíptica é atravessada pelo ducto comum da espermateca no sentido ventro-

Revista Brasileira de Zoologia 21 (2): 397-402, junho 2004 


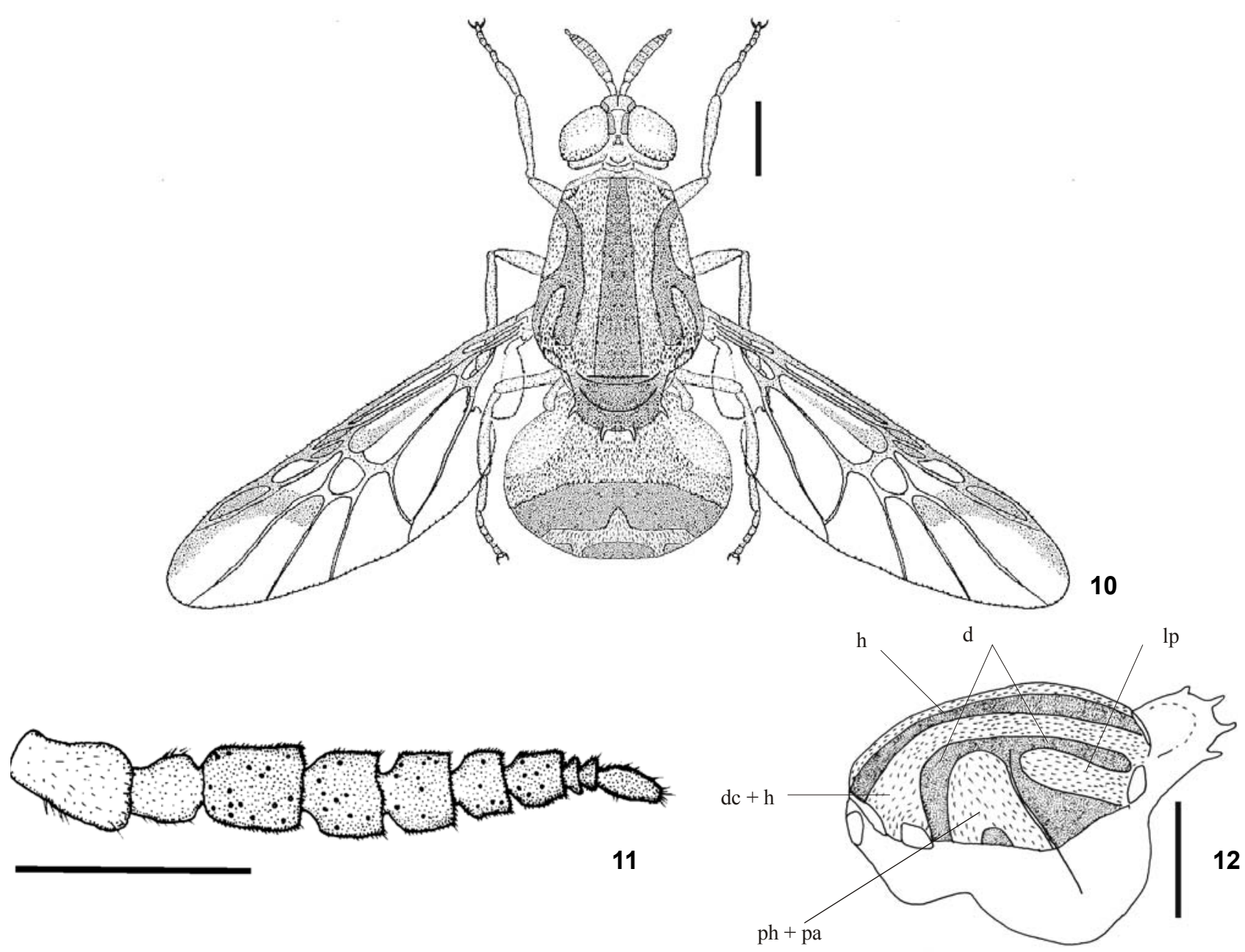

Figuras 10-12. Artemita podexargenteus fêmea: (10) habitus; (11) antena esquerda, vista lateral; (12) tórax em vista dorsolateral. (d) Dorsal, (dc) dorsocentral, (h) humeral, (lp) longitudinal posterior, (m) mediana, (pa) pré-alar, (ph) póshumeral. Escalas: 10 e $12=$ $1,0 \mathrm{~mm} ; 11=0,02 \mathrm{~mm}$.

dorsal, processo horizontal ausente, processos postero-laterais suavemente divergentes, pequenos e interligados por uma área pouco esclerotinizada e fortemente membranosa, que dificulta a visualização de seus limites (Fig. 17).

Espermatecas: dois ductos capsulares conectados a duas válvulas também conectadas a dois ductos expulsores incompletos e um ducto comum; os ductos capsulares são delgados, corrugados, com a base ligeiramente mais larga, a superfície atravessada por canalículos glandulares irregulares e com glândulas filiformes, mais numerosas no ápice do ducto; válvulas em forma de cotovelo ductos expulsores, na parte próxima à válvula estriados horizontalmente, revestidos por uma espessa camada membranosa translúcida, na parte próxima ao ducto comum, membranosos e inconspícuos (Figs 18-19); ducto comum, largo, membranoso e atravessando a abertura mediana da furca no sentido ventro-dorsal.
Material examinado: Brasil, Amapá: Serra do Navio, VIVII-1957, K. Lenko leg., 1 fêmea (MZSP); VIII-1959 Bicelli leg., 1 fêmea (MZSP). Pará: Mangabeira, Mocajuba, VII-1953, Orlando Rego leg., 1 fêmea (MZSP); Exp. Perm. Amaz. leg., 2-VIII-1968, Almeirim, 1 fêmea (MZSP); Paraquequara, 1 fêmea (MZSP); Barreirinha, Rio Tapajós, 10-XI-1970, 1 fêmea (MZSP). Amazonas: Tabatinga, IV-1958, E. Sousa Lima leg., 1 fêmea (MZSP). Rondônia: XI-1960, M. Alvarenga leg., 1 fêmea (DZUP). Mato Grosso: Maracajú, IV-1937, Serviço Febre Amarela leg., 1 fêmea (MZSP); Ponta Esperança, III-1944, Olala leg., 1 fêmea (MZSP); "Chapada", (300 m), Ponce Lane leg., 1 fêmea (MZSP). Espírito Santo: Córrego do Itá, X-1954, W. Zikan leg., 1 fêmea (MZSP); XI-XII-1956, 8 fêmeas (MZSP); Baixo Guandú, X-1946, P.C. Elias leg., 1 macho (MZSP). Rio de Janeiro: Seropédica, Universidade Federal Rural do Rio de Janeiro leg., IV-1959, 1 fêmea (MZSP); Itaguaí, I-XII1957, José Paulo leg., 1 fêmea (MNRJ). São Paulo: Araçatuba, 


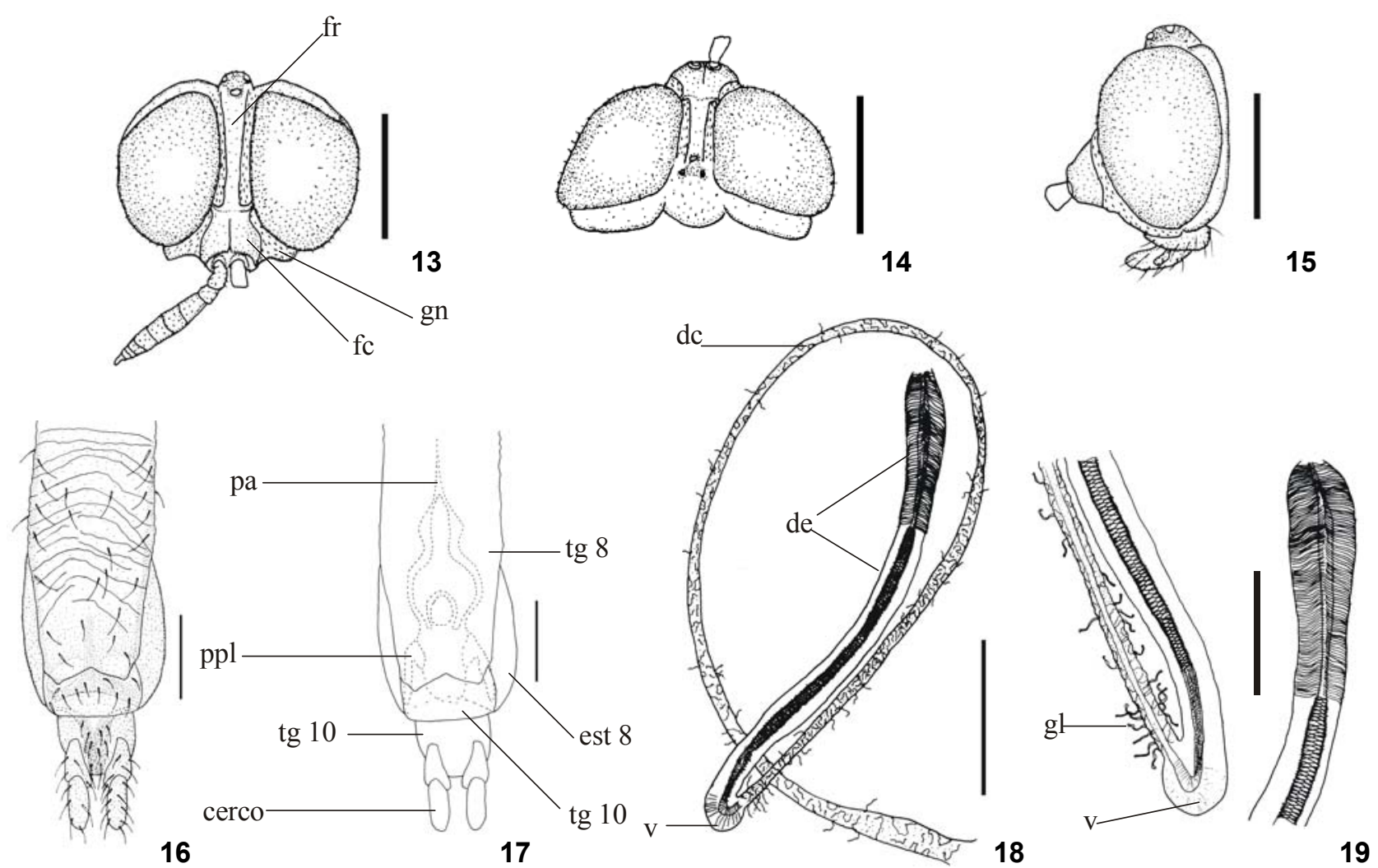

Figuras 13-19. Artemita podexargenteus fêmea. (13-15) Cabeça: (13) vista frontal; (14) vista dorsal; (15) vista lateral; (16-17) terminália: (16) vista dorsal; (17) esquema da terminália e furca, vista dorsal; (18-19) Espermateca: (18) ducto capsular, vávula e porção proximal do ducto expulsor; (19) detalhe do ducto expulsor e da válvula. (dc) Ducto capsular, (de) ducto espulsor, (est 8) oitavo esternito, (fc) face, (fr) fronte, (gn) gena, (gl) glândulas, (pa) processo anterior, (ppl) processo postero-lateral, (tg 8) oitavo tergito, (tg 9) nono tergito, (tg 10) décimo tergito, (v) válvula. Escalas: 13, 14 e $15=1,0 \mathrm{~mm} ; 16$ e $17=0,2 \mathrm{~mm} ; 18$ e $19=0,05 \mathrm{~mm}$.

Córrego Azul, II-1946, Barreto leg., 1 macho, 10 fêmeas (MZSP); Itaporanga, N.B. Antonina, I-1946, Barreto leg., 6 fêmeas (MZSP); Juquiá, I-1950, F. Lane leg., 2 fêmeas (MZSP). Paraná: Marechal Cândido Rondon (24운 34', 500 m), 5-III-1952, F. Plaumann leg., 1 fêmea (MZSP). Santa Catarina: Nova Teutônia (Seara, $27^{\circ} 11^{\prime}$ 's, 52 23E', 300-500 m), F. Plaumann leg., X-1944, 1 fêmea (MZSP); 01-X-1965, 10 machos, 10 fêmeas (MZSP); X-1971, 1 fêmea e 1 macho (MZSP); I-1972, 1 fêmea (MZSP); Nova Teutônia (Seara, $27^{\circ} 11^{\prime}$ S, 52 $23 E^{\prime}, 300-500$ m), F. Plaumann leg., VIII-1944, 1 fêmea (MZSP); X-1948, 1 fêmea e 1 macho (MZSP); VIII-1952, 1 fêmea (MZSP); XII-IX-1955, 1 fêmea (MZSP); X-1964, 1 fêmea (MZSP); I-X-1965, 1 fêmea (MZSP) IX-1967, 1 fêmea (MZSP).

Registros de distribuição geográfica: Argentina, Bolívia, Brasil (Amapá, Amazonas, Espírito Santo, Mato Grosso, Pará, Rondônia, Rio de Janeiro, São Paulo, Santa Catarina), El Salvador, México, Nicarágua, Panamá, Paraguai, Suriname, Tobago, Trinidad, Venezuela.

Comentários: de acordo com KeRTÉsz (1914) e JaMEs (1971), as espécies de Artemita apresentam o tórax com sete faixas for- madas por pêlos com cores diferentes, distribuídas sobre o mesonoto (Fig. 12): uma mediana ímpar (média), e seis pares dispostas dorso-lateralmente: dorso-centrais (dc); humerais $(\mathrm{h})$; pós-humerais (ph); pré-alares (pa) e longitudinais-posteriores (lp) e dorsais (d). O formato e a coloração destas faixas associados à coloração da mancha abdominal foram utilizados por estes autores para identificar as espécies de Artemita, principalmente $A$. podexargenteus, $A$. bicolor que estão segregadas umas das outras com base nos seguintes caracteres $-A$. podexargenteus: com faixas do mesonoto dorso-centrais, humerais, pós-humerais, préalares e longitudinais posteriores predominantemente prateadas ou com pêlos dourados na área posterior, logo após a sutura transversal; faixas dorsais e média pretas; mancha abdominal prateada; A. bicolor: com faixas do mesonoto dorso-centrais, humerais, pós-humerais, pré-alares e longitudinais posteriores predominantemente prateadas ou com pêlos dourados na área posterior, logo após a sutura transversal; faixas dorsais e média com pêlos cor de bronze; mancha abdominal prateada.

No material examinado, foi encontrada variação entre os

Revista Brasileira de Zoologia 21 (2): 397-402, junho 2004 
caracteres cromáticos das faixas do tórax usados para separar $A$. podexargenteus de $A$. bicolor, como discutido por JAMEs (1971). Não foram observadas diferenças no padrão de distribuição e fusão das faixas do mesonoto entre machos e fêmeas. $\mathrm{O}$ exame dos componentes da terminália masculina e feminina entre as duas espécies mostrou formas idênticas, apesar das variações cromáticas encontradas nas faixas do tórax, o que confirmou a opinião de JAMES (1971) de que A. bicolor e A. podexargenteus fossem sinônimos. Um exame do material tipo das duas espécies é inviável, uma vez que os síntipos de A. bicolor foram destruídos no incêndio do Museu de História Natural da Hungria em 1956 (Dr. Lásló Papp, com. pes.). O holótipo de $A$. podexargenteus, uma fêmea procedente de Santa Catarina, está depositado no Museu do Instituto de Zoologia da Academia de Ciências da Polônia. Ambos os nomes envolvidos nesta questão foram propostos no mesmo ano, sendo que o trabalho de ENDERLEIN (1914) foi publicado em sete de janeiro e o de KerTész (1914) em vinte de junho, deixando claro que a prioridade deve ser aplicada ao nome A. podexargenteus (ICZN 1999).

A dificuldade em se identificar corretamente as espécies do gênero Artemita com base em caracteres cromáticos é agravada pela descoloração post mortem e aponta para a necessidade de que sejam estabelecidos padrões morfológicos que, em acréscimo aos usuais, possam trazer bases seguras para a diferenciação das espécies do gênero. A análise das terminálias das espécies brasileiras do gênero Artemita possibilitou a observação de caracteres úteis para a identificação das espécies.

Com relação as espermatecas, a grande esclerotinização dos segmentos abdominais resultou num tempo maior de exposição aos reagentes utilizados para diafanização, o que determinou a perda de algumas estruturas mais sensíveis, entretanto foi possível observar e descrever em todas as dissecções as seguintes estruturas: dois ductos capsulares conectados a duas válvulas também conectadas dois ductos expulsores incompletos e um ducto comum.

\section{AGRADECIMENTOS}

Á CAPES pela bolsa de estudos concedida, através do Curso de Pós-Graduação em Biologia Animal da Universidade Federal Rural do Rio de Janeiro. Ao Prof. Dr. José Roberto PujolLuz pela orientação, estímulo e amizade. Às Profs Dra. Márcia Souto Couri (MNRJ), Dra. Eliana Marques Cancello (MZSP) e ao Prof. Dr. Cláudio José Barros de Carvalho (DZUP) pelo empréstimo dos exemplares das coleções de suas instituições. Aos Profs Dr. Francisco Racca Filho e Dr. Roberto de Xerez (UFRRJ), pelo apoio, sugestões e revisão deste manuscrito.

\section{REFERÊNCIAS BIBLIOGRÁFICAS}

Bigot, J.M.F. 1879. Dipterès nouveaux ou peu connus, $11^{\circ}$ Partie. XVI. Curiae Xilophagidarium et Stratiomydarum (Bigot) [Part]. Annales de la Société Entomologique de France, Paris, 9: 183-208.

Brauer, F. 1882. Die zweiflugler des Kaizerlichen Museums zu
Wien. II. Denkschriften der Kaiserlichen Akademie Mathematisch - Naturwissenchaftliche Classe Wien, Berlin, Cl. 44 (1): 59- 110.

Enderlein, G. 1914. Dipterologische Studien. VIII. Zur Kenntis der Stratiommyiden.Unter Familien mit 2ästiger Media Pachygasterinae, Lophotelinae und Prosocrhysinae. Zoologischen Anzeiger, Leipzig, 43: 289-315.

IczN. 1999. International Code of Zoological Nomeclature. London, International Trust for Zoological Nomeclature, $4^{\text {th }}$ ed., $365 p$.

JAMES, M.T. 1967. A preliminary review of the Argentine genera and species of Stratiomyidae (Diptera). Part 2. Pachygasterinae. Acta Zoologica Lilloana, Tucumán, 21: 95-121.

James, M.T. 1971. The South American species of Artemita. Jounal of the Kansas Entomological Society 44: 59-70. 1973. A catalog of the Diptera of the Americas south of the United States. 26. Family Stratiomyidae. São Paulo, Museu de Zoologia da Universidade de São Paulo, 95p.

James, M.T., M.W. McFadden \& N.E. Woodley. 1980. The Pachygastrinae (Diptera, Stratiomyidae) of middle America. Melanderia, Washington, 34 (2): 1-36.

KerTész, K. 1914. Vorarbeiten zu einer Monographie der Notacanthen. XXIV. Über die Gattung Artemita Walk. sens. lat. Annales historico-naturales Musei Nationalis Hungarici 12 (2): 449-557.

Lindner, E. 1928. Die von Prof. Dr. A. Seitz in Brasilien gesammenlten Stratiomyiden (Ins. Dipt.) Senkenbergiana, Frankfurt, 10 (6): 235-244.

Loew, H. 1860. Die Dipteren-Fauna Südafrika's. Erste Abtheilung. Mittler \& Sohn, Berlin I-XI, p. 1-330.

McAlpine, J.F.; B.V. Peterson; G. E. Shewell; H.J. Teskey; J.R. Vockroth \& D.M. Wood. 1981. Manual of Neartic Diptera. Ottawa, Research Branch, Agriculture Canada, vol. I, I-VI+674p.

Ururahy-Rodrigues, A.; J.R. Pujol-Luz \& P. Iide. 2000. Notas sobre a espermateca de Hermetia illucens (L., 1758) (Diptera, Stratiomyidae). Contribuições avulsas sobre a História Natural do Brasil, Série Zoologia, Seropédica, 17: 1-5.

WALKER, F. 1849. List of the specimens of Diptera insects in the collection of the British Museum. London, vol. 3, p. 485-697. .1854. List of the specimens of dipteras insects in the collection of the British Museum. London, Part. 5, p. 1-330.

Woodley, N.E. 1987. The Afrotropical Pachygastrinae genera Ashantina Kertéz and Meristomenigina James, with two new generic synonyms (Diptera, Stratiomyidae). Proceedings of the Entomological Society of Washington 89 (1): 103-121.

Woodley, N. E. 2001. A World Catalog of the Stratiomyidae (Insecta, Diptera). Myia, Leiden, 11: 1-475.

Recebido em 10.X.2003; aceito em 11.VI.2004. 